\title{
ON THE SPECTRUM OF $\psi$-CONTRACTING OPERATORS
}

\author{
ANWAR A. AL-NAYEF \\ Mu'tah University \\ Department of Mathematics and Statistics, PO Box 7 \\ Mu'tah, Al-Karak, Jordan \\ E-mail: anwar@mutah.edu.jo
}

(Received September, 1999 Revised August, 2000)

The spectrum $\sigma(A)$ of a continuous linear operator $A: E \rightarrow E$ defined on a Banach space $E$, which is contracting with respect to the Hausdorff measure of noncompactness, is investigated.

Key words: Contracting Operators, Measure of Noncompactness, Spectrum.

AMS subject classifications: $47 \mathrm{~A} 10$.

\section{Introduction}

Recall that a compact operator $A: E \rightarrow E$ defined on a Banach space $E$ is a continuous operator which maps every bounded set of $E$ into a relatively compact set. Equations involving compact operators arise naturally in the study of differential equations, integral equations and in many other applications. The spectrum of a compact operator $A$ in a Banach space $E$ has a simple structure analogous to that of an operator in a finite-dimensional space. It consists of at most a countable set, each element of the spectrum is an eigenvalue, and its only possible accumulation point is 0 (see Theorem 5.5-G, [9]). In particular, the number of eigenvalues of a compact operator outside any given circle centered at the origin in the complex plane is finite.

However, various applications of functional analysis involve operators which are not compact, i.e., operators which map bounded sets into sets with non-compact closure, for example, in the theory of delay differential equations of neutral type [2]. Some of these operators reduce the 'degree' of non-compactness, which in turn can be measured by different types of measure of non-compactness, such as Hausdorff measure of non-compactness and Kuratowski measure of non-compactness. For more information about different types of measures of noncompactness, see [1, Chapter 1]. A more general example of non-compact operators are condensing operators with respect to a specific measure of non-compactness $\psi$, and the $\chi$ - $\psi$-contracting operators, where $0<\chi<1$. It was proved in [7] that the spectrum of a $\chi$ - $\psi$-contracting operator $A$ (where $\psi$ here denotes the Kuratowski measure of non-compactness) outside the circle 
$|z|>2\|A\|^{\psi}$ (see the next section for the definition of $\psi$-norm) looks very similar to the spectrum of a compact operator. In this paper, we give a direct proof to the fact that the spectrum of a given $\chi$ - $\psi$-contracting operator, where $\psi$ is the Hausdorff measure of non-compactness, outside any circle of the form $|\lambda|>\chi+\varepsilon$ consists only of eigenvalues with finite multiplicities and the number of such values is finite. Definitions and some properties of condensing and contracting operators will be given in the next section. The main result and some lemmas will be given in Section 3. Section 4 will be devoted to the proof of the main theorem.

\section{Condensing Operators and Contractions}

Let $E$ be a Banach space with the norm $\|\cdot\|_{E}$. The Hausdorff measure of non-compactness $\psi(M)$ of a nonempty bounded subset $M$ of $E$ is defined by

$$
\psi(M)=\inf \{r>0: M \text { can be covered by finitely many balls of radius } r\} \text {. }
$$

Note that $\psi(M)=0$ if and only if $M$ is relatively compact. Some properties of $\psi$ that follow from the definition include the following:

(i) monotonicity: if $M_{1} \subseteq M_{2}$, then $\psi\left(M_{1}\right) \leq \psi\left(M_{2}\right)$;

(ii) sub-additivity: $\psi\left(M_{1}+M_{2}\right) \leq \psi\left(M_{1}\right)+\psi\left(M_{2}\right)$;

(iii) semi-additivity: $\psi\left(M_{1} \cup M_{2}\right)=\max \left\{\psi\left(M_{1}\right), \psi\left(M_{2}\right)\right\}$;

(iv) homogeneity: $\psi(k M)=|k| \psi(M), \quad k \in \mathbb{R}$.

Here $M, M_{1}$ and $M_{2}$ are nonempty bounded subsets of $E$. These properties are taken from [1] (see also [3]). For the following important property, see [1, Theorem 1.1.5].

(v) $\quad \psi(\operatorname{conv}(M))=\psi(M)$, where $\operatorname{conv}(M)$ denotes the closed convex hull of $M$.

Let $X$ be a subset of $E$. A continuous mapping $f: X \rightarrow E$ is said to be $\psi$-condensing on $X$ if

$$
\psi(f(M))<\psi(M)
$$

whenever $M \subset X$ is bounded and not relatively compact. A condensing mapping $f$ on $X$ is called $\chi-\psi$-contracting on $X$, where $0 \leq \chi<1$, if

$$
\psi(f(M)) \leq \chi \psi(M)
$$

for all bounded subsets $M$ of $X$. Finally, the $\psi$-norm of the operator $A: E \rightarrow E$ is defined to be

$$
\|A\|^{\psi}=\inf \{k: M \subseteq E \text { bounded implies } A(M) \text { bounded and } \psi(A(M)) \leq k \psi(M)\}
$$

or equivalently [1],

$$
\|A\|^{\psi}=\sup \{\psi(A(M)): M \subseteq E \text { bounded and } \psi(M)=1\} .
$$

Clearly $\|A\|^{\psi}=0$ if and only if $A$ is compact. Some properties of the $\psi$-norm of the operator $A$ are listed in [1, Lemma 2.6.7]. 


\section{Main Result}

Recall from [1] that a continuous linear operator $B: E \rightarrow E$ is said to be a Fredholm operator if it satisfies the following properties:

(i) $\quad \alpha=\operatorname{dim} N(B)<\infty$, where $N(B)=\{x \in E: B x=0\}$;

(ii) $\overline{B E}=B E$, (where the overline denotes closure);

(iii) $\beta=\operatorname{codim} B E<\infty$;

(iv) the number ind $B=\beta-\alpha$, called the index of $B$, is equal to zero.

The Fredholm spectrum $\sigma_{\Phi}(B)$ of the operator $B$ is the set of complex numbers $\lambda$ such that the operator $B-\lambda I$ is not a Fredholm operator. Set $R_{\Phi}(B)=\sup \{|\lambda|$ : $\left.\lambda \in \sigma_{\Phi}(B)\right\}$.

We mention the following result of [1] which involves the spectral theory of Fredholm operators

Theorem 1: [1] Let $E$ be a Banach space and $A: E \rightarrow E$ a continuous linear operator. Then for each complex number $\lambda$ with $\lambda>R_{\Phi}(A)$, the operator $A-\lambda I$ is a Fredholm operator of index zero.

A detailed statement about the spectral properties of a $\chi$ - $\psi$-contracting operator with $\chi<1$ is given in the following main result of this paper.

Theorem 2: Let $E$ be a Banach space and $A: E \rightarrow E$ a continuous linear operator which is $\chi$ - $\psi$-contracting on $E$ with $\chi<1$. For each $\varepsilon>0$, let

$$
\sigma_{\varepsilon}(A)=\{\lambda \in \sigma(A):|\lambda|>\chi+\varepsilon\}
$$

Then

(i) each $\lambda \in \sigma_{\varepsilon}(A)$ is an eigenvalue of $A$;

(ii) the set $\sigma_{\varepsilon}(A)$ is finite;

(iii) each $\lambda \in \sigma_{\varepsilon}(A)$ is of finite multiplicity.

The proof of this theorem requires the following lemmas.

Lemma 1: (Riesz's Lemma) Let $D$ be a closed proper subspace of the normal space $E$ and let $\rho \in(0,1)$. Then there exists a unit vector $z \in E$ such that $\|x-z\|_{E} \geq \rho$ for all $x \in D$.

The proof of the following lemma follows that of Lemma 5.2 in [7] with a slight modification, so it will be omitted.

Lemma 2: Let $A: E \rightarrow E$ be a continuous linear operator which is $\chi$ - $\psi$-contracting with $\chi<1$ and let $\lambda \in \sigma_{\varepsilon}(A)$. If $A-\lambda I$ is injective, then $A-\lambda I$ is surjective and $(A-\lambda I)^{-1}$ is continuous and linear.

The following two lemmas are standard results, see Theorem 7.4-3 in [6] and Proposition 9.6 in [3].

Lemma 3: Let $\left\{\lambda_{k}: k \geq 1\right\}$ be a set of distinct eigenvalues of the operator $A: E \rightarrow E$. For each $k \geq 1$, let $x_{k}$ be a nonzero eigenvector corresponding to $\lambda_{k}$. Then the set $\left\{x_{1}, x_{2}, \ldots, x_{k}\right\}$ is linearly independent for each $k \geq 1$.

Lemma 4: Let $A$ be a continuous linear operator on a Banach space $\left(E,\|\cdot\|_{E}\right)$. Then, for every $\tau>0$, there exists an equivalent norm $\|\cdot\|_{\tau}$ on $E$ such that $\|A\|_{\tau} \leq r(A)+\tau$, where $r(A)$ denotes the spectral radius of $A$. 


\section{Proof of Theorem 2}

Proof of (i): Let $\lambda \in \sigma_{\varepsilon}(A)$ be given and suppose that it is not an eigenvalue of $A$. Then the operator $A-\lambda I$ is injective and by Lemma 2 , the operator $(A-\lambda I)^{-1}$ is continuous, that is, $\lambda$ cannot belong to the continuous spectrum of $A$. By Lemma 2 again, the operator $(A-\lambda I)^{-1}$ is surjective and hence the range of $A-\lambda I$ is dense in $E$, that is, $\lambda$ cannot belong to the residual spectrum of $A$. This contradicts the fact that the spectrum of an operator consists of three disjoint sets: eigenvalues, continuous spectrum and residual spectrum. Therefore $\lambda$ must be an eigenvalue of $A$.

Proof of (ii): Suppose that $\sigma_{\varepsilon}(A)$ is infinite, and let $\left\{\lambda_{k}\right\}_{k=1}^{\infty}$ be any sequence of distinct eigenvalues of $A$ taken from $\sigma_{\varepsilon}(A)$. For each $k \geq 1$, let $x_{k}$ be a nonzero eigenvector corresponding to the eigenvalue $\lambda_{k}$. By Lemma 3 , the set $\left\{x_{i}: i=1,2, \ldots, k\right\}$ is linearly independent. If $M_{k}$ denotes the linear subspace of $E$ spanned by $\left\{x_{i}: i=1,2, \ldots, k\right\}$, then $M_{k-1}$ is a closed, proper subspace of $M_{k}$. Let $\rho$ be such that $\frac{\chi}{\chi+\varepsilon}<\rho<1$. By Lemma 1 , there exists a sequence $\left\{z_{k}\right\}_{k=1}^{\infty}$ of unit vectors such that $z_{k} \in M_{k}$ and $\left\|x-z_{k}\right\| \geq \rho$ for all $x \in M_{k-1}$. Note that $\left\|z_{k}-z_{k-1}\right\| \geq \rho$, and hence

$$
\rho \leq \psi\left[\left\{z_{k}: k \geq 1\right\}\right] \leq 1
$$

Let $x \in M_{k}$, then $x=\sum_{i=1}^{k} a_{i} x_{i}$, where $a_{i}$ is a constant for every $i=1,2, \ldots, k$, we also have

$$
A x=\sum_{i=1}^{k} a_{i} A x_{i}=\sum_{i=1}^{k} a_{i} \lambda_{i} x_{i}
$$

and

$$
\left(A-\lambda_{k} I\right) x=\left(A-\lambda_{k} I\right) \sum_{i=1}^{k} a_{i} x_{i}=\sum_{i=1}^{k} a_{i} \lambda_{i} x_{i}-\sum_{i=1}^{k} a_{i} \lambda_{k} x_{i}=\sum_{i=1}^{k-1} a_{i}\left(\lambda_{i}-\lambda_{k}\right) x_{i} .
$$

This shows that

$$
A M_{k} \subset M_{k} \text { and }\left(A-\lambda_{k} I\right) M_{k} \subset M_{k-1}
$$

Note that $\left(A z_{j}-\lambda_{j} z_{j}\right) \in M_{j-1}$ and $A z_{k} \in M_{k}$ imply $\left(A z_{j}-\lambda_{j} z_{j}\right)-A z_{k} \in M_{j-1}$ $-M_{k}$. It follows that for $j>k \geq 1$, we have $M_{j-1}-M_{k} \subset M_{j-1}$ and hence

$$
\begin{gathered}
\left\|A z_{j}-A z_{k}\right\|=\left\|\left(A z_{j}-\lambda_{j} z_{j}-A z_{k}\right)+\lambda_{j} z_{j}\right\| \\
=\left|\lambda_{j}\right|\left\|-\lambda_{j}^{-1}\left(A z_{j}-\lambda_{j} z_{j}-A z_{k}\right)-z_{j}\right\| \\
\geq\left|\lambda_{j}\right| \rho \geq(\chi+\varepsilon) \rho .
\end{gathered}
$$

Thus by inequality (4.1) and property (iii) of $\psi$, we have

$$
\psi\left[A\left(\left\{z_{k}: k \geq 1\right\}\right)\right] \geq(\chi+\varepsilon) \rho>\chi \geq \chi \psi\left[\left\{z_{k}: k \geq 1\right\}\right]
$$


which contradicts the fact that $\psi\left[A\left(\left\{z_{k}: k \geq 1\right\}\right)\right] \leq \chi \psi\left[\left\{z_{k}: k \geq 1\right\}\right]$.

Proof of (iii): Since the set $\sigma_{\varepsilon}(A)$ is finite, it is sufficient to show that the geometric multiplicity of the generalized eigenspace $E^{k}$ corresponding to a fixed eigenvalue $\lambda_{k}$ of the set $\sigma_{\varepsilon}(A)$ is finite. For each $\delta>0$, let

$$
B\left(\lambda_{k}, \delta\right)=\left\{z \in \mathbb{C}:\left|z-\lambda_{k}\right|<\delta\right\}
$$

Choose $\delta>0$ and $\varepsilon>0$ such that $B\left(\lambda_{k}, \delta\right) \cap B\left(\lambda_{j}, \delta\right)=\emptyset$ for $j \neq k$, and $B\left(\lambda_{k}, \delta\right) \cap$ $\{z \in \mathbb{C}:|z| \geq \chi\} \neq \emptyset$. This is possible since $\sigma_{\varepsilon}(A)$ is finite. So the spectrum $\sigma(A)$ of $A$ consists of two disjoint sets $\sigma(A)=\left\{\lambda_{k}\right\} \cup\left(\sigma(A) \backslash\left\{\lambda_{k}\right\}\right)$. By the Decomposition Theorem [4, Theorem 6.17 , p. 178], the space $E$ can be decomposed into a direct sum

$$
E=E_{k}^{-} \oplus E_{k}^{+}
$$

such that $E_{k}^{-}$and $E_{k}^{+}$are invariant under $A$ and the spectrum $\sigma\left(\left.A\right|_{E_{k}^{-}}\right)$of the restriction is $\left\{\lambda_{k}\right\}$; whereas, the spectrum $\sigma\left(\left.A\right|_{E_{k}}\right)$ is $\sigma(A) \backslash\left\{\lambda_{k}\right\}$. The theorem also ensures the existence of bounded projections $P^{-}$of $E$ onto $E_{k}^{-}$and $P^{+}$of $E$ onto $E_{k}^{+}$.

Let $c=\frac{\left|\lambda_{k}\right|+\chi}{2}$ and consider the operator $\tilde{A}:=\frac{1}{c} A$. For any bounded subset $M$ of $E$ with $\psi(M)>0$, we have

$$
\psi[\tilde{A}(M)]=\frac{1}{c} \psi(A(M)) \leq \frac{\chi}{c} \psi(M),
$$

and hence the operator $\tilde{A}$ is $\left(\frac{\chi}{c}\right)-\psi$-contracting with $\frac{\chi}{c}<1$. The spectrum of the restriction $\left.\tilde{A}\right|_{E_{k}^{-}}$consists of one point, namely

$$
\sigma\left(\left.\tilde{A}\right|_{E_{k}^{-}}\right)=\frac{1}{c} \sigma\left(\left.A\right|_{E_{k}^{-}}\right)=\frac{\lambda_{k}}{c}
$$

Note also that the inverse $\left.\tilde{A}\right|_{E_{k}} ^{-1}$ exists with spectral radius satisfying

$$
r\left(\tilde{A} \mid E_{k}^{-1}\right)=\frac{c}{\left|\lambda_{k}\right|}<1 .
$$

By Lemma 4, for each $\tau>0$ there exists an equivalent norm $\|\cdot\|_{\tau}$ in $E$ such that

$$
\left\|\tilde{A} \mid \bar{E}_{k}^{-1}\right\|_{\tau} \leq \frac{c}{\left|\lambda_{k}\right|}+\tau
$$

Choose $\tau>0$ small enough such that $\left\|\left.\tilde{A}\right|_{E_{k}} ^{-\frac{1}{}}\right\|_{\tau}<1$; hence, the operator $\left.\tilde{A}\right|_{E_{k}}$ is expanding in the new norm $\|\cdot\|_{\tau}$.

Let $U$ be the unit ball in $E_{k}^{-}$. Since $\left.\tilde{A}\right|_{E_{k}^{-}}$is expanding, then $\left.U \subseteq \tilde{A}\right|_{E_{k}^{-}}(U)$ and thus

$$
\psi(U) \leq \psi\left[\left.\widetilde{A}\right|_{E_{k}^{-}}(U)\right] \leq \frac{\chi}{c} \psi(U) .
$$

Note that we have used the same symbol $\psi$ here to represent the new Hausdorff measure of non-compactness defined by means of the new equivalent norm $\|\cdot\|_{\tau}$. It follows from inequality $(4.2)$, that $\psi(U)=0$ since $\frac{\chi}{c}<1$. This means that $U$ is compact and hence the space $E_{k}^{-}$is finite-dimensional. This completes the proof of (iii) and hence the theorem. 
Remark: It was shown in Proposition 5.8 of [7], that for $r \geq 0$, if $\sigma_{r}(A)=\{\lambda \in$ $\sigma(A):|\lambda| \geq r\}$ and if $r>2\|A\|^{\alpha}$, then $\sigma_{r}(A)$ is finite (or empty) and each member of $\sigma_{r}(A)$ is an eigenvalue of $A$. Here $\alpha$ denotes the Kuratowski measure of non-compactness which is defined for the set $M$ as

$$
\alpha(M)=\inf \{r>0: M \text { can be covered by finitely many sets of diameter }<r\},
$$

and $\|A\|^{\alpha}$ denotes the $\alpha$-norm of $A$. Our result differs from this result in that it is valid for any spectral value $|\lambda|>\chi$.

\section{Acknowledgements}

The author would like to thank Professor Peter Kloeden and Professor Alexei Pokrovskii for their useful suggestions and Professor Boris Sadovskii for his comments. The author is also grateful for the suggestions of an anonymous referee which have improved the readability of the paper considerably.

\section{References}

[1] Akhmerov, R.R., Kamenskii, M.I., Potapov, A.S., Rodkina, A.E. and Sadovskii, B.N., Measure of Non-compactness and Condensing Operators, Birkhäuser Verlag 1992. (Trans. from Russian by A. Jacob).

[2] Al-Nayef, A.A., Kloeden, P.E. and Pokrovskii, A., Semi-hyperbolic mappings, condensing operators and neutral delay equations, J. Diff. Eqns. 137 (1997), 320-339.

[3] Deimling, K., Nonlinear Functional Analysis, Springer-Verlag, New York 1985.

[4] Kato, T., Perturbation Theory for Linear Operators, second edition, SpringerVerlag, New York 1976.

[5] Krasnosel'skii, M.A. and Zabreiko, P.P., Geometrical Methods of Nonlinear Analysis, Springer-Verlag, New York 1984.

[6] Kreyszig, E., Introductory Functional Analysis with Applications, John Wiley and Sons, New York 1978.

[7] Martin, R.H., Nonlinear Operators and Differential Equations in Banach Spaces, John Wiley and Sons, New York 1976.

[8] Sadovskii, B.N., Limit-compact and condensing operators, Russ. Math. Surveys 27 (1972), 85-155.

[9] Taylor, A.E., Introduction to Functional Analysis, John Wiley and Sons, New York 1958. 


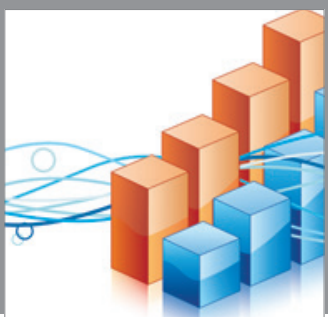

Advances in

Operations Research

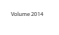

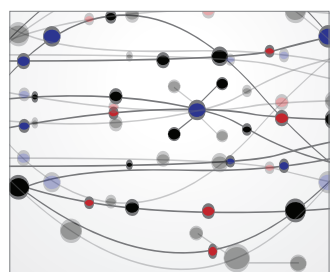

\section{The Scientific} World Journal
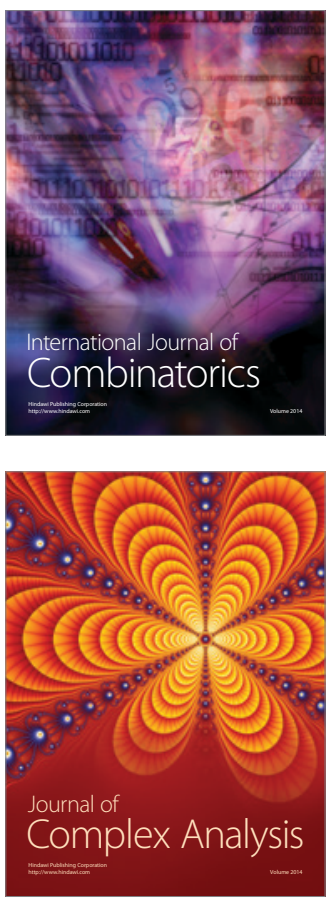

International Journal of

Mathematics and

Mathematical

Sciences
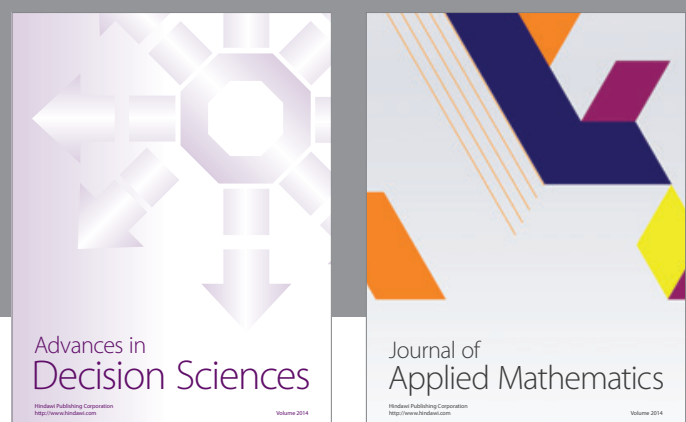

Journal of

Applied Mathematics
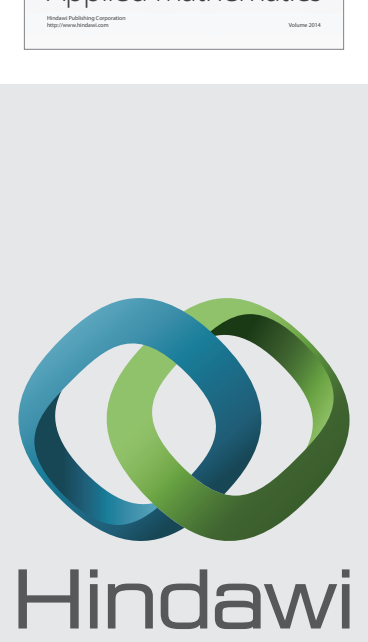

Submit your manuscripts at http://www.hindawi.com
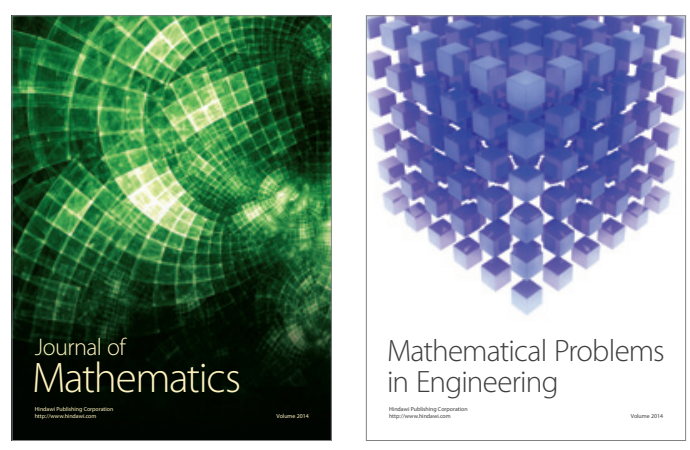

Mathematical Problems in Engineering
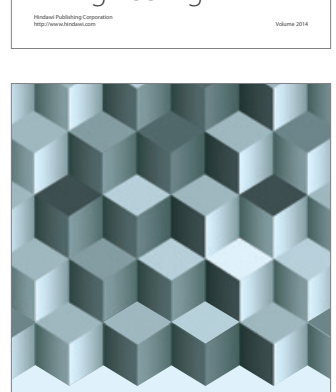

Journal of

Function Spaces
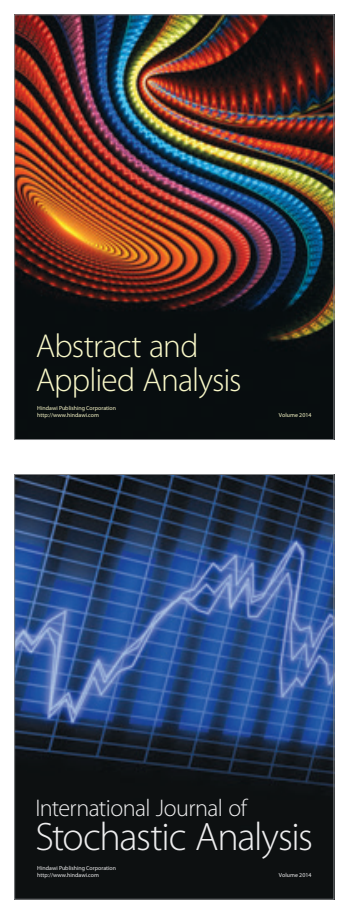

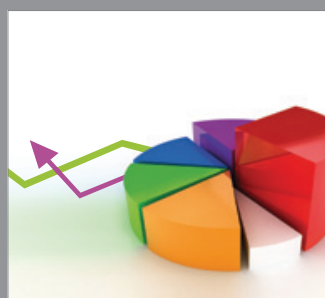

ournal of

Probability and Statistics

Promensencen
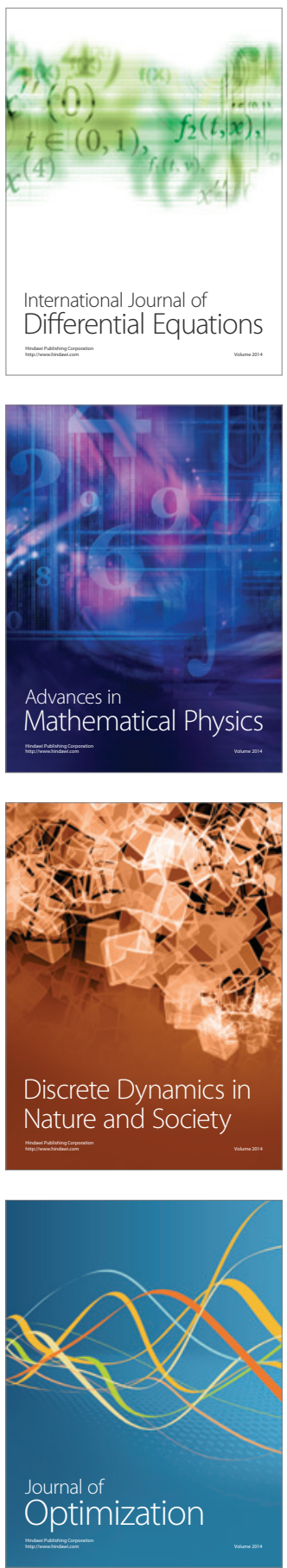